\title{
Un programme proposé pour conscientiser les enseignants du FLE autour de la dyslexie
}

\section{Dr. Chaïmaa Mohamed Tawfik Mohamed}

Maître de conférences de didactique du FLE - Département de curricula \& méthodologie- Faculté de Pédagogie Université d'Alexandrie.

\section{Résumé}

$e$ but ultime de cette étude était d'éveiller la
conscience des enseignants du FLE autour de la
notion de la dyslexie. En vue d'atteindre ce but, la chercheuse a élaboré deux outils: un programme proposé aux enseignants et un pré- post test autour de la dyslexie. Quant au programme, il était destiné à douze enseignants du FLE inscrits au diplôme général de l'année académique 2015/ 2016. Il leur présentait, de manière plus ou moins exhaustive, la dyslexie: sa définition, ses causes, ses répercussions, quelques aménagements pédagogiques censées amoindrir les conséquences néfastes de la dyslexie, etc. Afin de tester l'efficacité de ce programme à conscientiser les enseignants du FLE à propos de la dyslexie, la chercheuse a appliqué un pré-post test sur le groupe en question tout en ayant recours au «test $W$ des signes et rangs» de Wilcoxon pour deux échantillons appariés. Les résultats de l'étude montrent clairement que les différences entre les notes des enseignants au pré-post-test sont significatives en faveur du post-test, ce qui met en exergue l'effet positif qu'exerce ce programme sur la conscience des enseignants du FLE autour de la dyslexie.

Mots clés: Troubles de langage, dyslexie, conscience, enseignants du FLE.

\section{Introduction}

Depuis de longues années, de nombreux pays ont accordé, par respect aux droits de l'homme, un intérêt particulier aux troubles d'apprentissage qui représentent l'un des problèmes majeurs qu'affrontent certains élèves. D'après l'Association canadienne des troubles d'apprentissage (ACTA) (2002), ce concept désigne "un certain nombre de dysfonctionnements pouvant affecter l'acquisition, l'organisation, la rétention, la 
compréhension ou le traitement de l'information verbale ou non verbale. Ces dysfonctionnements affectent l'apprentissage chez des personnes qui, par ailleurs, font preuve des habiletés intellectuelles moyennes essentielles à la pensée ou au raisonnement". En effet, cette incapacité significative et persistante résultant d'une déficience ou d'une inaptitude empêche les élèves d'accomplir normalement des activités quotidiennes (Conseil national pour l'éducation spécialisée, 2014, p. 10). Or, il est important de souligner que ces troubles sont nombreux, parmi lesquels citons la dyslexie, la dysphasie, la dyscalculie, etc.

D'ailleurs, dans la présente étude, la chercheuse abordera la dyslexie considérée comme un trouble durable du langage écrit ayant un impact négatif non seulement sur la lecture, mais aussi sur l'orthographe et l'écriture. De nos jours, un nombre croissant d'élèves souffre de la dyslexie et de ses conséquences néfastes, très souvent à l'insu des parents ou des enseignants. Il est vraisemblable que ce manque de conscience, dû éventuellement à une méconnaissance de la nature de ce trouble, de ses répercussions et de l'importance d'un dépistage précoce et d'une rééducation propice, constitue non seulement un frein majeur à l'apprentissage scolaire, mais également un véritable risque de dégradation psychologique et de désocialisation à l'âge adulte des dyslexiques. Ceux-ci entreraient par la suite dans la spirale de l'échec à tous les niveaux. C'est pourquoi Hunziker et Pulzer-Graf (2012, p. 12) affirment qu'il paraît indispensable de conscientiser les enseignants autour de la dyslexie afin de procurer, au dyslexique, une aide et un soutien adéquats tout en assurant sa scolarisation dans un environnement le plus «normal» et le moins restrictif possible (Hunziker et Pulzer-Graf, 2012, p. 12).

\section{Problématique de l'étude}

La problématique de l'étude actuelle réside en un manque de conscience, de la part d'un bon nombre d'enseignants du FLE, de la nature de la dyslexie. En fait, ceux-ci considèrent toute difficulté de lecture accompagnée d'une mauvaise écriture comme faisant partie de la dyslexie. En d'autres termes, ils sont 
incapables de distinguer les vrais dyslexiques des faux dyslexiques. De surcroît, ils sont incapables de doter les apprenants en question d'une rééducation propice sous prétexte qu'un tel ajustement sera inutile.

\section{On peut formuler cette problématique à partir des trois questions suivantes:}

1. Quel est le degré de conscience des enseignants du FLE autour de la dyslexie?

2. Quel est le programme proposé afin de développer la conscience des enseignants du FLE autour de la dyslexie?

3. Quelle est l'efficacité du programme suggéré?

\section{Objectifs de l'étude}

La présente étude vise à:

1. conscientiser les enseignants du FLE sur la nature de la dyslexie en tant que trouble d'apprentissage durable et persistant.

2. développer chez les enseignants une posture réflexive visà-vis des difficultés que peuvent affronter leurs élèves lors de l'apprentissage de la lecture.

3. familiariser les enseignants à une multitude de pistes de rééducation censées alléger les conséquences néfastes de la dyslexie.

4. aider les dyslexiques eux - mêmes, via des aménagements pédagogiques propices, à récupérer leurs faiblesses en lecture sans se perdre dans la spirale de l'échec.

\section{Importance de l'étude}

L'étude actuelle acquiert son importance du fait qu'elle:

1. aide les enseignants à assurer un enseignement qui tente d'assouvir les besoins particuliers de leurs apprenants dyslexiques.

2. constitue la première étude égyptienne, à notre connaissance, visant à développer la conscience des enseignants du FLE sur la dyslexie. 
3. attire l'attention des responsables pour prendre en considération l'importance de procurer aux enseignants des ateliers de formation qui visent à développer leurs compétences professionnelles afin d'aider et de soutenir pédagogiquement les élèves ayant des troubles d'apprentissage.

\section{Limites de l'étude}

Il s'agit de/du/d':

1. douze enseignants du FLE inscrits au diplôme général à la faculté de Pédagogie d'Alexandrie durant l'année académique 2015/ 2016.

2. un programme proposé dont le but est de développer la conscience des enseignants autour de la dyslexie.

3. "test $\mathrm{W}$ des signes et rangs» de Wilcoxon pour deux échantillons appariés" comme technique statistique permettant d'examiner le niveau de conscience des enseignants autour de la dyslexie.

\section{Hypothèse de l'étude}

Il y a une différence statistique significative entre la moyenne des rangs concernant les notes des enseignants du FLE au pré-post-test portant sur la dyslexie en faveur du post-test.

\section{Terminologie de l'étude}

La dyslexie est un trouble d'apprentissage de la lecture et de l'orthographe d'origine neurologique qui se manifeste non seulement par des difficultés à reconnaître les mots, mais également par des faibles compétences en orthographe et en décodage chez des personnes intelligentes, indemnes de tout déficit sensoriel et de tout problème psychologique et qui vivent dans des milieux favorables d'un point de vue économique, social et culturel.

\section{Le cadre théorique}

En effet, la dyslexie constitue l'un des sujets les plus controversés autour duquel il ne s'agit point d'unanimité scientifique. Ceci renvoie, selon Ringard (2000), à deux raisons 
de natures différentes: premièrement, une raison sémantique selon laquelle les définitions représentent soit le champ étiologique, soit le champ sémiologique, soit les deux à la fois. Deuxièmement, une raison conceptuelle ou idéologique où l'on considère la dyslexie tantôt comme un trouble neurologique, tantôt comme un trouble spécifique de l'apprentissage de la lecture.

Dans ce qui suit, la chercheuse tentera de présenter une esquisse de quelques-unes de ces définitions car une recension exhaustive de celles-ci dépasse le but ultime de cette étude.

1. D'un point de vue étymologique, la dyslexie est un mot d'origine grecque composé du préfixe "dys" qui signifie mauvais, difficulté ou trouble et de "lexie" qui signifie mot (Doan, 2014; Denis, 2009, p. 12). Selon le dictionnaire français l'Internaute ${ }^{1}$ : la dyslexie est un nom féminin signifiant un "trouble de la lecture, (une) difficulté à reproduire le langage écrit".

2. L'Institut national de la santé de l'enfant et du développement de l'homme (Cité par Martin et Borkowski, 2014, p. 20), Lyon, Shaywitz et Shaywitz (2003) et L'Association internationale de la dyslexie à Ontario (2002): La dyslexie est un trouble spécifique d'apprentissage d'origine neurobiologique qui renvoie à des difficultés concernant la reconnaissance et l'orthographe des mots aussi bien que des capacités limitées du décodage des mots.

3. Augade et Thyss (2000):"Troubles spécifiques de l'apprentissage de la lecture qui apparaissent chez des enfants d'une intelligence normale, sans aucun déficit sensoriel et poursuivant une scolarité conventionnelle".

4. L'Association canadienne de la dyslexie2: "C'est une difficulté que certaines personnes éprouvent avec l'alphabet, la lecture, l'écriture et l'épellation, en dépit d'une intelligence moyenne ou supérieure à la moyenne, de

$1 \mathrm{http}: / /$ www.linternaute.com/dictionnaire/fr/definition/dyslexie/.

2 http://www.dyslexiaassociation.ca/francais/questce.shtml. 
méthodes d'enseignement conventionnelles et d'une influence positive du milieu socioculturel. La dyslexie est probablement d'origine génétique et héréditaire".

A la lueur des définitions mentionnées ci-dessus, on constate que la dyslexie est d'origine neurologique et héréditaire, elle atteint des personnes intelligentes n'ayant aucun déficit sensoriel. De même, elle n'a aucun rapport ni avec les méthodes traditionnelles d'enseignement, ni avec la bassesse des niveaux économique, social et culturel du dyslexique et de ses parents.

\section{Des mythes courants autour de la dyslexie}

Or, afin de mieux cerner ce sujet équivoque, il s'avère essentiel de présenter les principaux mythes répandus parmi les non- spécialistes tout en clarifiant en quoi sont- ils erronés. Parmi ces mythes citons ( Thorwarth, 2014, p. 52; Golliet, 2011, p. 13; Arseneault et al., 2009, p. 4; Brazeau-Ward, 2003, p. 1-2):

1. La dyslexie est rare: En effet, la dyslexie constitue, selon l'Institut national de la santé mentale aux Etats-Unis, le trouble d'apprentissage le plus courant.

2. Les dyslexiques ne peuvent pas réussir dans la vie. Au contraire, ce trouble d'apprentissage n'a pas empêché Léonard de Vinci, Albert Einstein, Agatha Christie, Jules Verne et Auguste Rodin d'avoir une grande notoriété par leurs chez- d'œuvres dans divers domaines. Il est simplement question de déployer plus d'efforts.

3. La dyslexie est un trouble difficile à diagnostiquer. Il est vraisemblable que le diagnostic de la dyslexie, comme toute autre chose, est facile lorsqu'on sait ce que l'on cherche.

4. Les problèmes de lecture peuvent disparaître avec l'âge. Ce trouble persiste durant l'adolescence; le dyslexique, même après un long entraînement, ne lit pas de la même vitesse qu'un normal.

5. La dyslexie atteint les garçons plus que les filles. En effet, maintes études récentes ont prouvé qu'il n'y a aucune 
différence entre les deux sexes en ce qui concerne ce trouble.

6. La répétition d'une classe peut mettre terme à la dyslexie. En revanche, la dyslexie ne sera pas allégée que lorsqu'on se sert des méthodes adéquates et efficaces pour les cas dyslexiques.

7. L'inversement des graphèmes ou des chiffres est l'unique signe évocateur de la dyslexie. En fait, cet inversement peut se remarquer, à la fois, chez les dyslexiques et les non - dyslexiques surtout au début de l'apprentissage de l'écriture. De même, 10\% seulement des dyslexiques inversent les graphèmes en écrivant. Leur vrai problème réside en l'identification des lettres et non pas dans le copiage ou l'écriture de ces dernières.

8. Les parents qui ne font pas la lecture à leurs enfants sont la principale cause de la dyslexie. En effet, certains parents font souvent la lecture à leurs enfants, mais ceux ci sont dyslexiques.

9. La dyslexie est diagnostiquée chez un enfant dès le début de son apprentissage de la lecture. Néanmoins, de nombreux spécialistes affirment que le diagnostic doit être posé à partir du moment où l'écart entre l'âge chronologique de développement et l'âge de lecture est suffisamment significatif (plus que 18 mois).

10. Seuls les psychologues peuvent évaluer les personnes ayant la dyslexie. En effet, toute personne formée à ce type d'évaluation peut le faire.

\section{Une perspective historique}

Par ailleurs, en survolant l'histoire de la dyslexie au cours des années, on constate qu'elle a été connue vers la fin du XIXe siècle. En 1896, Morgan, un médecin anglais, décrivait, dans le journal intitulé British Médical, le cas d'un garçon qui souffrait d'un problème majeur dans l'apprentissage de la lecture et l'orthographe, tandis qu'il avait de très bonnes capacités intellectuelles. Quelques années plus tard, Hinshelwood, un ophtalmologiste, publia une monographie présentant des cas 
similaires d'enfants intelligents, mais incapables de lire. Ces différents cas mentionnés par Morgan et Hinshelwood étaient considérés comme des alexiques ayant une "cécité verbale congénitale" liée à un défaut des fonctions cérébrales concernant la mémoire visuelle des mots. Ils supposaient donc une origine neurologique à cette incapacité à lire. En revanche, le titre "alexique" n'était pas adéquat, puisqu'il est souvent utilisé afin de décrire ceux qui sont incapables de lire suite à un accident vasculaire cérébral. (Denis, 2009, p. 9- 12; Institut national de la santé et de la recherche médicale (INSERM), 2007, p. 419; Van Hout et Estienne, 2001, p. 21; Augade et Thyss, 2000, p. 7 et 8).

Vers le début du XXe siècle, des pédagogues commençaient à accorder plus d'intérêt à ce phénomène. En effet, ils le percevaient sous un autre angle en réfutant la conception médicale et précisément neurologique accordée à la dyslexie. Ils estimaient que les causes de la dyslexie sont multiples y compris: une pédagogie déficitaire, des troubles psychologiques, un manque de motivation, etc. Dans ce cadre, la dyslexie se révélait en tant qu'un type très grave de retard de lecture (Van Hout et Estienne, 2001, p. 21).

A partir des années 1920, de nombreux chercheurs et cliniciens américains ont examiné profondément les travaux européens portant sur les rapports entre le cerveau et le comportement d'enfants et d'adultes ayant des troubles d'apprentissage (Saint- Pierre, Dalpé, Lefebvre et Giroux, 2010, p. 98). A l'issue de cette examination, le neuropsychiatre et neuropathologiste américain Orton perçut non seulement l'intelligence des enfants dyslexiques, mais aussi leurs perceptions visuelles et visio-spatiales excellentes. Il souligna, de surcroît, une caractéristique fondamentale des dyslexiques, à savoir la perception inversée des graphèmes qu'il nomma "strephosymbolia" (Van Hout et Estienne, 2001, p.22). Celle-ci est considérée "comme faisant partie non d'une dysfonction cérébrale, mais d'une anomalie de dominance hémisphérique; dans ces conditions, l'hémisphère gauche perdrait la suprématie qu'habituellement il exerce sur le droit" (Van Hout et Estienne, 
2001, p. 22). Donc, à l'instar de Morgan et Hinshelwood, Orton supposait que la dyslexie est un problème neurologique; toutefois, il adoptait une position complètement différente. Pour lui, la dyslexie émanait d'une dominance cérébrale anormale plutôt qu'une lésion au niveau des fonctions cérébrales. De plus, Orton mit en relief le rôle prépondérant que joue l'hérédité dans la parution de la dyslexie surtout chez les garçons (Ibid.). A cet égard, il faut bien signaler que les théories d'Orton ainsi que ses méthodes de rééducation étaient favorablement accueillies par les pédagogues, ce qui n'était plus le cas avec les hypothèses neurologiques précédentes (Saint- Pierre et al., 2010, 98; Van Hout et Estienne, 2001).

Dans les années 70, une nouvelle ère commença avec les travaux de Vellutino qui affirmait que la dyslexie constitue une déficience cognitive de type phonologique. En d'autres termes, la dyslexie n'est que le résultat d'un déficit à établir les liens graphèmes- phonèmes indispensables pour la lecture. En effet, au cours des 30 dernières années, maintes recherches ont été conduites et elles ont toutes confirmé les constatations de Vellutino (Snowling, 2013, p. 7; Valdois, 2005, p. 268).

\section{Les Théories explicatives de la dyslexie}

A la lueur du panorama historique présenté ci-dessus, il s'avère clairement que maintes études ont été accomplies autour de la dyslexie et en particulier sur le fonctionnement particulier de la structure cérébrale d'un dyslexique. Evidemment, l'hémisphère droit du dyslexique réalise des tâches effectuées par l'hémisphère gauche chez un non-dyslexique. A titre d'illustration, chez un non- dyslexique, l'hémisphère gauche du cerveau est chargé de contrôler partiellement les fonctions du langage, de l'écriture et de la lecture (Juhel, 1998, p. 140). Mais, dans le cas d'un dyslexique, lorsque l'hémisphère droit "se charge de remplir cette fonction, il y a confusion et distorsion au niveau de l'interprétation des signaux envoyés au cerveau lorsque l'enfant lit. (...) Donc, chez l'enfant dyslexique, la lecture est déficiente à cause d'une incapacité cérébrale lors de la réception 
des messages; dans le cas de l'écriture, le blocage se fait lors de la transmission du message" (Juhel, 1998, p. 140).

Mais la question qui se pose maintenant: pourquoi le cerveau d'un dyslexique fonctionne t-il différemment d'un cerveau standard? En effet, la réponse à cette question est à l'origine de nombreux débats qui ne sont pas encore résolus. De surcroît, ce débat a donné naissance, au cours des années, à de nombreuses théories visant à expliquer ce phénomène, parmi lesquelles citons:

1. La théorie visuelle: D'un point de vue historique, cette théorie fut la première à interpréter la dyslexie. Les adeptes de cette théorie présumaient que les erreurs commises par les dyslexiques, lors de la lecture, renvoient essentiellement au dysfonctionnement des voies magnocellulaires de leur système visuel. Ils ajoutaient que la vision du dyslexique pourrait être normale et toutefois, il confond des graphèmes qui se ressemblent d'un point de vue physique tels que ( $m$ et $n / i$ et l, etc.) et d'autres qui diffèrent par la symétrie selon leur axe vertical ( $p$ et $q$, $b$ et $d$ ), tout en éprouvant des difficultés d'ordre spatial face aux stimuli non verbaux (comme les dessins géométriques). En effet, ces confusions et ces difficultés proviennent essentiellement de la déficience des régions cérébrales responsables de la représentation spatiale des objets visuels (Sprenger-Charolles et Sernicales, 2003, p. 79; Habib, 2002, p. 10).

2. La théorie phonologique: les partisans de cette théorie postulent que la difficulté qu'éprouve un dyslexique à lire émane principalement d'un manque de conscience phonologique. Ceci se manifeste clairement par une déficience particulière au niveau de la représentation, du stockage et de la récupération des sons de la parole, ce qui aura un impact négatif sur l'apprentissage des correspondances graphème - phonème, étape préliminaire à l'apprentissage de la lecture. C'est 
pourquoi les tenants de cette théorie assurent que la conscience phonologique dont jouit l'enfant joue un rôle prépondérant à prédire son niveau en lecture ultérieurement (Dickie, Ota et Clark, 2013; Habib, 2002, p. 10, 11; Snowling, 2001).

3. La théorie du déficit du traitement temporel: Il s'agit d'un compromis entre les deux théories précédentes. Selon cette théorie, un enfant dyslexique est incapable de distinguer non seulement les sons brefs, mais également les éléments non linguistiques s'ils se suivent rapidement. Ces troubles phonologiques et visuels dont souffre le dyslexique se référent au dysfonctionnement du traitement des aspects temporels particuliers à chacune des modalités sensorielles (Habib, 2014, p. 130; Chaix, Trabanino, Taylor et Demonet, 2005, p. 77; Habib, 2002, p. $10,11)$.

4. La théorie cérébelleuse: Les tenants de cette théorie constatent qu'un dysfonctionnement du cervelet est responsable de la parution d'une déficience concernant l'automatisation des capacités sensori-motrices chez les dyslexiques. C'est pourquoi les dyslexiques témoignent très fréquemment des troubles d'écriture (la dysgraphie) ainsi que des troubles articulatoires (L'association internationale de la dyslexie, 2012; Marin et Legros, 2008, p. 55).

En dépit de la diversité des théories explicatives de la dyslexie, celle-ci est reconnue, aujourd'hui d'une manière quasiunanime, comme le résultat d'un trouble phonologique empêchant d'acquérir les liens graphèmes- phonèmes fondamentaux à l'apprentissage de la lecture. En d'autres mots, la dyslexie constitue une forme particulière de trouble de langage (en tant que sous-type des troubles d'apprentissage) ayant un impact négatif sur la manière du cerveau à encoder les traits phonologiques des mots (Nijakowska, 2010, p. 4-5; Valdois, 2005). Selon Torgesen, Foorman et Wagner (2007, p.2), il est vraisemblable que les problèmes de traitement phonologique 
qu'éprouvent les dyslexiques interfèrent de façon négative significative sur le développement de la conscience phonologique indispensable pour l'acte de lire.

\section{Dyslexie ou dyslexies?}

Or, il s'agit de deux principaux types de dyslexie, à savoir la dyslexie acquise et la dyslexie développementale (Bessac, 2013, p.125, 126):

1. La dyslexie acquise: Elle paraît chez des personnes qui, à la suite d'un accident cérébral, sont brutalement devenus incapables de lire.

2. La dyslexie développementale: Elle survient au cours du développement cérébral des enfants normalement intelligents sous forme de troubles durables et sévères dans les processus d'acquisition de la lecture. Ces troubles se révèlent en dépit d'un enseignement adéquat, d'un niveau socio - économique convenable et de l'absence de tout déficit sensoriel, ce qui les oppose donc aux difficultés d'apprentissage. Il faut bien signaler que ce type de dyslexie émane du dysfonctionnement neurologique de certaines zones impliquées dans le processus de la lecture, alors que d'autres fonctionnent parfaitement.

A cet égard, il s'avère indispensable de signaler que ce qui nous intéresse dans cette présente étude est la dyslexie développementale. Celle-ci se subdivise, selon de nombreux auteurs, en trois sous-types (Lederlé et Maeder, 2014, p. 376, 377; Saint- Pierre et al., 2010, p. 107; Lapierre, 2008, p. 256; Pelletier et Léger, 2004; De Partz et Valdois, 2000, p. 783;):

1. La dyslexie phonologique (dysphonie): Elle constitue le sous - type le plus fréquent de la dyslexie développementale. Elle renvoie à un déficit spécifique au niveau de la voie indirecte ou d'assemblage. Celle-ci consiste, selon le modèle de Coltheart et Coll, à segmenter les mots en graphèmes, puis à correspondre chaque graphème à son phonème et à regrouper, enfin, les phonèmes pour accéder à la forme sonore du mot 
(Aghababian et Nazir, 2007: 84). Un dyslexique phonologique éprouve de grandes difficultés à automatiser des règles de conversion graphophonémiques indispensables pour lire des mots nouveaux. En revanche, il lit bien les mots connus parce qu'il les a mémorisés visuellement. Nous pouvons donc constater qu'il s'agit d'un déficit concernant le décodage des mots.

2. La dyslexie de surface (dyséidétique): Elle se révèle par des difficultés au niveau de la voie directe ou d'adressage. Cette dernière, d'après le modèle de Coltheart et Coll, concerne les mots connus. Elle permet d'accéder à leur connaissance globale à travers un lexique mental élaboré durant l'apprentissage de la lecture (Aghababian et Nazir, 2007: 84). Un enfant, ayant ce type de dyslexie, réussit à lire, mais lentement, les mots réguliers, Cependant, il affronte des problèmes majeurs lors de la lecture et de l'écriture des mots irréguliers. En effet, ceci provient du fait qu'il n'arrive pas à garder en mémoire les morphèmes qui facilitent une reconnaissance rapide du mot qu'il lit. Il souffre, de surcroît, d'une dysorthographie souvent plus sévère que chez les dyslexiques phonologiques. Afin de pallier ses difficultés, le dyslexique préfère utiliser la procédure de conversion de graphèmes en phonèmes. C'est pourquoi, un dyséidétique a tendance à rédiger les mots tels qu'ils se prononcent sans tenir compte de leurs spécificités orthographiques. A titre d'exemple, pour un dyséidétique, le mot "taxi" s'écrit "taqsi.

3. La dyslexie mixte: Il s'agit d'une combinaison des difficultés des deux types de dyslexie, mentionnés cidessus. Il est question donc non seulement de problèmes de traitement des sons, mais également d'un trouble de mémorisation des mots. A ces problèmes s'ajoutent plus de confusions spatiales.

A cet égard, il faut bien souligner que certains auteurs affirment que le type de dyslexie n'est pas fixe tout au long du 
développement de l'enfant. Par contre, il peut varier en fonction de l'âge (Chaix et al. 2005, p.75).

\section{Une constellation de troubles}

$\mathrm{Au}$ cours du processus de son apprentissage, un enfant dyslexique peut affronter d'autres troubles portant sur l'écriture, la parole, le calcul, etc. En fait, à l'absence d'un soin particulier adéquat, l'ensemble de ces troubles contribueront à freiner l'apprentissage scolaire de l'enfant tout en lui causant, à long terme, des problèmes psychiques (Golliet, 2011). Voici une présentation brève de quelques-uns de ces troubles.

1. La dysgraphie: est considérée comme dysgraphie "toute écriture qui, en absence de trouble neurologique avéré, empêche le scripteur de structurer sa pensée, de libérer ses émotions, de mettre en forme ses sentiments [...]. Ceci parce que son écriture n'est pas assez rapide pour suivre le rythme de la pensée, pas assez lisible pour qu'il puisse communiquer une idée à autrui ou même se relire luimême, parce qu'elle est une entrave à un développement scolaire normal" (Thoulon-Page, 2009, p.10). Ainsi, nous pouvons constater que dû à l'écriture très lente et illisible des dyslexiques, ils peuvent être considérés comme des dysgraphiques.

2. La dysphasie: La dysphasie est un déficit durable et permanant du langage. Elle se caractérise par des troubles sévères au niveau de la compréhension et/ou de l'expression du langage parlé, en l'absence de perte auditive, de déficience mentale, ou d'un trouble affectif (Leclercq et Leroy, 2012, p.5; Direction générale de l'enseignement obligatoire (DGEO), 2008, p. 7). Quant au rapport entre la dyslexie et la dysphasie, de nombreuses études récentes affirment que l'apprentissage du langage écrit et celui du langage oral sont intrinsèquement liés. Il est vraisemblable que l'aptitude à apprendre à lire dépend essentiellement des aptitudes phonologiques dont jouit l'enfant. Celles- ci consistent en sa capacité à manipuler les composantes sonores de la parole. Un 
dyslexique manifeste de grandes difficultés à effectuer certaines tâches phonologiques telles que le jugement de rimes, la suppression d'une syllabe ou d'un phonème, etc. D'ailleurs, un taux important de dyslexiques ont affronté des difficultés précoces au cours de leur apprentissage à parler. C'est pourquoi, de nombreux auteurs estiment que les troubles du langage oral et écrit appartiennent à un même continuum (Habib, Giraud, Rey et Robichon, 2003, p. 39).

3. La dyscalculie: C'est un trouble qui se rapporte à l'apprentissage des mathématiques y compris les opérations de calculs et la compréhension mathématique. Il faut souligner que souvent les dyslexiques sont bons en mathématiques, toutefois ils y sont pénalisés pour les difficultés majeures à savoir écrire, lire et assimiler les consignes (Golliet, 2011, p. 28).

4. La dyspraxie: Selon l'association québécoise pour les enfants dyspraxiques, la dyspraxie ou le trouble de l'acquisition de la coordination est "un trouble neurodéveloppemental qui affecte [la capacité de l'enfant] à planifier, à organiser et à automatiser les gestes moteurs pour réaliser une action ou une activité" 3 . Autrement dit, la personne conçoit les gestes mais n'arrive pas à les appliquer alors que ses fonctions motrices et sensorielles sont normales (Golliet, 2011, p. 26).

5. La dysorthographie: C'est un trouble spécifique et durable de l'acquisition de l'orthographe qui paraît chez un enfant qui ne présente aucun déficit sensoriel ou intellectuel. Un enfant dysorthographique rencontre des difficultés durables à comprendre et à appliquer les règles orthographiques au cours de l'écriture spontanée ou de l'écriture sous dictée. Par exemple, il a des difficultés majeures à effectuer la conversion phono - graphique, à segmenter les composantes d'une phrase, à mettre en œuvre les conventions orthographiques (les règles

$3 \mathrm{http}: / /$ www.dyspraxie-aqed.ca/dyspraxie-aqed_008.htm. 
d'usage) aussi bien que l'orthographe grammaticale sous forme de marques flexionnelles (l'accord et la conjugaison) (Bessac, 2013, p. 131, Délitroz et al., S.D., p. 6). De nombreux auteurs postulent que la dysorthographie et la dyslexie sont intimement liées l'un à l'autre. En effet, la dysorthographie n'est qu'une conséquence directe du trouble phonologique clairement remarqué chez les dyslexiques (Bessac, 2013, p.131). C'est pourquoi d'aucuns affirment que tout dyslexique est forcément dysorthographique (Golliet, 2011, p. 26).

Or, à partir de ce survol des différents types de dyslexie aussi bien que son rapport très étroit avec d'autres troubles, il s'avère indispensable de conscientiser les enseignants autour de la nature de la dyslexie, de ses signes révélateurs et de ses incidences afin de garantir, au dyslexique, une aide et un soutien propices. A cet égard, il faut bien souligner que depuis de longues années, de nombreuses études ont été élaborées pour dépister les dyslexiques et les prendre en charge tout en évitant le retentissement de ce trouble sur l'apprentissage scolaire en général. Parmi ces études citons à titre d'exemple:

Etude d'Ortiz (2014): Cette étude visait à améliorer les performances des élèves dyslexiques au niveau de la production écrite à travers un entraînement à la planification. Pour atteindre cet objectif, la chercheuse a mis en œuvre un protocole expérimental auprès d'élèves dyslexiques et d'autres ayant des difficultés sévères en production écrite. Les résultats montrent l'effet significatif de l'entraînement à la planification sur certaines variables de la production écrite.

Etude de Dejonckère (2013): Cette étude a proposé, aux enseignants, diverses pistes pédagogiques censées aider les dyslexiques à surmonter les difficultés de compréhension. Via cette étude, la chercheuse a constaté que les adaptations pédagogiques suggérées sont efficaces non seulement pour les 
langues mais également pour les autres disciplines où la lecture s'y avère primordiale.

Etude de Zorman, Lequette et Pouget (2004): Cette étude accomplie par l'équipe du Laboratoire Cogni- Sciences de l'IUFM de l'Académie de Grenoble en collaboration avec le service de santé scolaire avait pour but de favoriser l'intégration, à l'école et dans la société, des enfants présentant des facteurs de risque d'échec en lecture. Pour réaliser ce but, les chercheurs ont élaboré et étalonné un outil de dépistage des enfants en question. Cet outil a joué un rôle prépondérant à doter l'enseignant des activités de prévention pédagogiques, d'adaptation et de remédiation aussi bien que des prises en charge rééducatives, tout en permettant au médecin scolaire de désigner les enfants qui ont besoin d'un suivi régulier et de juger ceux qui doivent être adressés pour un bilan approfondi.

Il est incontestable que ces études destinées directement aux dyslexiques ou aux enseignants travaillant auprès de ceux ci visaient principalement, via une multitude d'aménagements pédagogiques, à améliorer les performances de ces élèves et à les aider à éviter la situation d'échec. Ce qui met en évidence l'importance d'accomplir, dans notre société égyptienne, de nombreuses études afin de conscientiser les enseignants et les parents autour de la dyslexie et des diverses pistes pédagogiques supposées amoindrir l'impact négatif de la dyslexie sur la vie de l'enfant.

\section{Méthodologie de l'étude}

Dans la présente étude, la chercheuse a pris appui tant sur la méthode descriptive que sur la méthode quasi expérimentale. Le recours à la méthode descriptive était pour élaborer le cadre théorique autour de la dyslexie aussi bien que le programme destiné aux enseignants. Quant à la méthode quasi - expérimentale, elle nous a permis d'identifier l'impact qu'exerce le variable indépendant (sous forme de programme suggéré) à développer le variable dépendant, à savoir la conscience des enseignants du FLE autour de la dyslexie. 
Cette partie de l'étude se subdivise en les sous - parties suivantes:

1. L'échantillon: L'échantillon de l'étude a été sélectionné parmi les enseignants inscrits au diplôme général, section française de l'année académique 2015/ 2016. Ils étaient au nombre de douze enseignants. L'âge moyen des enseignants interrogés était de 28.6 ans. L'expérience dans l'enseignement du FLE, dont jouit l'échantillon de l'étude, varie comme suit: quatre ont enseigné pour cinq ans, trois pour trois ans, deux pour deux ans, deux pour un an et un pour huit ans. Or, huit de ces enseignants travaillent auprès des enfants du petit primaire (de la première à la troisième), tandis que quatre enseignent à ceux de la maternelle. A cet égard, il faut bien souligner que ces enseignants travaillent auprès d'enfants dont l'âge est critique. Autrement dit, c'est à partir de la maternelle qu'on peut prévenir, via les difficultés très sévères qu'éprouve l'enfant, qu'il serait dyslexique ou non. Néanmoins, on ne peut pas confirmer cette prétention que lorsqu'il sera en deuxième primaire. En outre, il est important de noter qu'on ne peut pas généraliser les résultats auxquels on aboutira sur l'ensemble des enseignants du FLE. Le cas échéant, l'on est mené à déduire qu'il ne s'agit pas d'un échantillon représentatif des caractéristiques de toute la population vu le nombre limité des participants. Or, il faut bien noter que les noms des participants à cette étude sont symbolisés par des lettres de l'alphabet afin de garder la confidentialité de l'expérience scientifique.

2. Outils de l'étude: La présente étude est fondée sur l'usage de deux outils: Un pré-post test autour de la dyslexie et un programme suggéré aux enseignants. Voici une présentation plus ou moins détaillée de chacun de ces outils. Commençons par le pré-post test. Celui- ci est préparé par la chercheuse afin de déterminer le niveau de la conscience des enseignants autour de la dyslexie. Cet 
outil renferme vingt six questions variées entre: mettez vrai ou faux (en justifiant votre réponse), cochez la bonne réponse, complétez, distinguez, à l'aide de preuves claires et nettes, les écrits des vrais dyslexiques de ceux des faux dyslexiques et proposez des interventions pédagogiques pour aider l'enfant dyslexique. La note totale du test est soixante points distribuée comme suit: la question de vrai ou faux est sur vingt points: un point pour le signe correct et un point pour la justification de la réponse. Quant à la question de cocher la bonne réponse, il est sur cinq points: un point est attribué à chaque réponse correcte. La question de compléter est sur quinze points: trois points sont réservés à chaque phrase. Pour les questions de proposer des interventions pédagogiques et celles de distinguer les écrits des dyslexiques de ceux des non dyslexiques, il s'agit de dix points pour chacune. De plus, la chercheuse a élaboré un modèle de réponse pour le pré- post test de l'étude.

En ce qui concerne le programme proposé, il a pour but de conscientiser les enseignants du FLE autour de la dyslexie. Il se compose de trois séances de deux heures chacune. La première séance introduit les troubles d'apprentissage tout en les différenciant des difficultés d'apprentissage. Elle présente également la définition de la dyslexie en tant que l'un des troubles d'apprentissage des langues, ses types, ses causes aussi bien que quelques exemples de personnes douées dans différents domaines en dépit de leur dyslexie. La deuxième séance traite, en premier lieu, les signes révélateurs de la dyslexie en lecture, en écriture, à l'écoute, en mémoire, etc. En second lieu, cette séance aborde le dépistage de la dyslexie. La séance en question s'achève par une activité qui permet aux enseignants du FLE de s'entraîner à distinguer les écrits des dyslexiques de ceux des non dyslexiques. Quant à la troisième séance, elle est consacrée aux aménagements pédagogiques conformes aux besoins particuliers des dyslexiques dans divers aspects de leur apprentissage. Tout au long de ce programme, la chercheuse a mis l'accent sur le rôle crucial que joue l'enseignant au dépistage 
précoce des dyslexiques. Elle a souligné également l'importance de la collaboration de l'enseignant avec les parents et les spécialistes pour aider le dyslexique, via des moyens propices, à surmonter quelques unes de ses problèmes sévères.

\section{Déroulement}

En vue de déterminer l'efficacité du programme proposé pour développer la conscience des enseignants du FLE autour de la dyslexie, la chercheuse a suivi les étapes suivantes. Durant le deuxième semestre de l'année académique 2015/ 2016, les enseignants ont été interrogés, dans un premier temps, sur la dyslexie par l'intermédiaire d'un pré-test. Dans un deuxième temps, la chercheuse a appliqué, durant trois semaines, le programme suggéré sur l'échantillon de l'étude dans le but de le familiariser avec la notion de dyslexie ainsi que la prise en charge d'un dyslexique. Dans un troisième temps, la chercheuse a appliqué le post-test qui a duré 90 minutes à l'instar du pré-test. Par la suite, la chercheuse s'est servie du «test $\mathrm{W}$ des signes et rangs» de Wilcoxon pour deux échantillons appariés.

\section{Méthode d'analyse des données}

La chercheuse a eu recours au "test $\mathrm{W}$ des signes et rangs» de Wilcoxon pour deux échantillons appariés", en tant que protocole de recherche quantitative qui convient parfaitement au nombre restreint de l'étude actuelle. En effet, ce test non paramétrique met en exergue le niveau de différence entre les notes de l'échantillon de l'étude au pré-post-test ${ }^{4}$. Ce qui nous permet donc d'investiguer l'effet qu'exerce le programme suggéré par la chercheuse sur le degré de conscience des enseignants du FLE autour de la dyslexie.

\section{Les résultats}

A la lueur des résultats de l'échantillon de l'étude au prétest, on dénote leur faible degré de conscience autour de la dyslexie. Ceci semble évident via leurs fausses réponses à la

${ }^{4}$ http://www.xlstat.com/fr/centre-d-apprentissage/tutoriels/12.html. 
majorité des questions proposées par le test en question et l'incapacité de justifier leurs réponses aux questions de vrai ou faux et notamment à celle de distinguer les écrits des vrais dyslexiques des non-dyslexiques. De surcroît, il faut bien souligner que leurs réponses, concernant les interventions pédagogiques qu'ils proposent aux dyslexiques, étaient très générales de type: "lui donner des cours supplémentaires, varier les méthodes d'explication, etc.", ce qui prouve un manque de conscience clair et net de la nature de la dyslexie et des besoins spécifiques des élèves dyslexiques.

En analysant les réponses de ces enseignants, la chercheuse a découvert que pour la plupart d'entre eux la dyslexie constitue un problème d'apprentissage dû à un retard mental sans qu'ils ne sachent ni les causes, ni les conséquences. De plus, certains estimaient que toute intervention pédagogique auprès des dyslexiques s'avère inutile.

Quant aux résultats des enseignants du FLE au post-test, on remarque, grosso modo, une amélioration dans leurs notes. Ce progrès se manifeste clairement par l'intermédiaire de plusieurs éléments parmi lesquels citons: leurs réponses correctes à la majorité des questions, leurs justifications plausibles à de nombreuses questions, leurs capacités de distinguer les écrits des dyslexiques de ceux des non-dyslexiques, aussi bien que leurs suggestions pertinentes à la question concernant les interventions pédagogiques réclamées pour les dyslexiques. Ce qui prouve que le programme proposé a contribué au développement de leur conscience autour de la dyslexie. Le tableau (1) et la figure (1) mettent en relief le progrès dans les notes des enseignants (dans le post-test par rapport à celles du pré-test. 
Tableau (1) Les notes des enseignants au pré-post-test concernant la dyslexie

\begin{tabular}{|l|l|l|}
\hline Enseignants du FLE & Pré- test & Post-test \\
\hline A & 14 & 50 \\
\hline B & 10 & 40 \\
\hline C & 13 & 47 \\
\hline D & 16 & 48 \\
\hline F & 11 & 55 \\
\hline G & 13 & 46 \\
\hline H & 11 & 42 \\
\hline I & 15 & 57 \\
\hline J & 13 & 51 \\
\hline K & 13 & 53 \\
\hline L & 13 & 48 \\
\hline & 15 & 58 \\
\hline
\end{tabular}

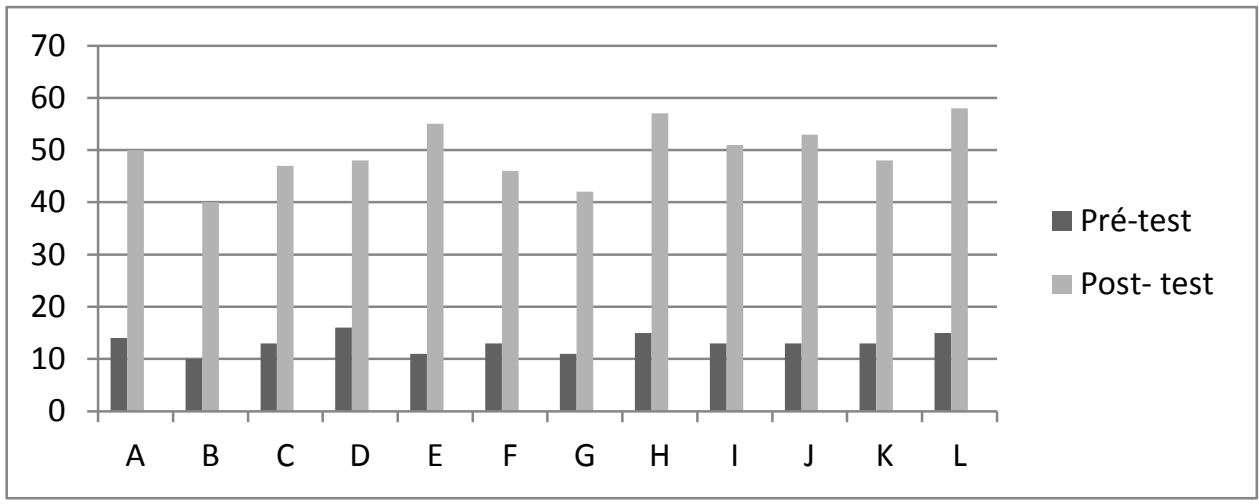

Figure (1) Les notes des enseignants au pré-post-test concernant la dyslexie

A partir du tableau (1) et de la figure (1), la chercheuse a constaté que, dans le pré-post test, les notes des participants à l'étude n'ont pas été influencées par leurs années d'expérience. En d'autres mots, l'expérience limitée de l'enseignant ne reflète pas nécessairement son manque de conscience autour du sujet de la dyslexie et vice versa. A titre d'illustration, l'enseignant (B) qui a huit ans d'expérience dans le domaine de l'enseignement du FLE a témoigné moins de conscience que son collègue (L) qui a trois ans d'expérience, tant au pré-test qu'au post-test. Nous pouvons donc noter que la diversité des années d'expérience n'exerce aucun effet sur les résultats de cette étude. 
Or, en vue d'expliquer les différences entre les notes des enseignants au pré-post-test concernant la conscience autour de la dyslexie, il semble nécessaire de calculer le test de Wilcoxon pour les différentes questions du test. Le tableau suivant illustre l'application du test de Wilcoxon en fonction des deux tests prédéterminés.

Tableau (2): Application du test de Wilcoxon sur les notes des enseignants au pré-post test concernant leur conscience autour de la dyslexie

\begin{tabular}{|c|c|c|c|c|c|c|}
\hline & $\begin{array}{l}\text { Enseignants } \\
\text { du FLE }\end{array}$ & $\begin{array}{l}\text { Pré- } \\
\text { test }\end{array}$ & $\begin{array}{l}\text { Post- } \\
\text { test }\end{array}$ & $\begin{array}{l}\text { Différences } \\
\text { entre les } \\
\text { notes au } \\
\text { pré-post- } \\
\text { test }\end{array}$ & $\begin{array}{l}\text { Somme des } \\
\text { rangs des } \\
\text { différences } \\
\text { de signe } \\
\text { "plus" } \\
\text { (P) }\end{array}$ & $\begin{array}{l}\text { Somme des } \\
\text { rangs des } \\
\text { différences } \\
\text { de signe } \\
\text { "moins" } \\
\text { (M) }\end{array}$ \\
\hline & A & 14 & 50 & 36- & - & 7- \\
\hline & B & 10 & 40 & 30- & - & 1- \\
\hline & C & 13 & 47 & -34 & - & 5- \\
\hline & D & 16 & 48 & -32 & - & 3- \\
\hline & E & 11 & 55 & -44 & - & 12- \\
\hline & $\mathbf{F}$ & 13 & 46 & -33 & - & 4- \\
\hline & G & 11 & 42 & -31 & - & $2-$ \\
\hline & $\mathrm{H}$ & 15 & 57 & -42 & - & 10 \\
\hline & I & 13 & 51 & -38 & - & 8- \\
\hline & $\mathbf{J}$ & 13 & 53 & -40 & - & -9 \\
\hline & K & 13 & 48 & -35 & - & 6- \\
\hline & L & 15 & 58 & -43 & - & 11- \\
\hline Total & & & & & & 78- \\
\hline
\end{tabular}

\section{A partir de ce tableau, on constate que/ qu':}

1. La valeur de $(\mathrm{M})$ est inférieure à celle mentionnée dans le tableau statistique de Wilcoxon, ce qui indique que les différences entre les notes au pré-post-test sont significatives en faveur du post-test.

2. Les scores obtenus lors du post-test mettent donc en lumière le développement de la conscience des enseignants en ce qui concerne la dyslexie, ce qui prouve l'efficacité du programme élaboré.

3. Un développement de la conscience des enseignants autour de la dyslexie, sa signification, ses causes, ses signes évocateurs, son impact négatif sur l'apprentissage 
en général, aussi bien que la manière de distinguer les vrais dyslexiques des non-dyslexiques s'avère donc indispensable pour les enseignants du FLE. En effet, cette constatation convient parfaitement avec celle réclamée par l'étude de Knudsen (2012) et celle de Felber et Michel (2012).

\section{Conclusion}

En guise de conclusion, il est incontestable que cette étude a été accomplie en vue de conscientiser les enseignants du FLE à propos de l'un des plus importants troubles d'apprentissage qu'ils peuvent fréquenter, à savoir la dyslexie. En vue de réaliser ce but, la chercheuse a élaboré un programme adressé aux enseignants du FLE inscrits au diplôme général qui travaillaient auprès des élèves aux cycles maternel et primaire. L'efficacité de ce programme a été identifiée à l'aide d'un pré-post test tout en se servant du modèle statistique de Wilcoxon. Les résultats de la présente étude montrent clairement que les différences entre les notes des enseignants au pré-post-test sont significatives en faveur du post-test. Ce qui prouve l'efficacité du programme suggéré à développer la conscience des enseignants du FLE à propos de la dyslexie. En effet, la prise de conscience que doit manifester l'enseignant face aux troubles d'apprentissage, et en particulier la dyslexie, est indéniable pour l'aider à sélectionner des stratégies, des techniques et des activités en concordance avec les besoins particuliers de ces élèves.

\section{Recommandations}

$\mathrm{Vu}$ le nombre restreint de l'échantillon de l'étude actuelle, la chercheuse recommande l'achèvement de nombreuses études avec un grand nombre de sujets afin de pouvoir, d'une part confirmer les résultats auxquels elle a abouti, et d'autre part les généraliser pour en profiter ultérieurement.

La chercheuse recommande, d'emblée, l'accroissement des cours et des ateliers de formation destinés aux enseignants du FLE, surtout au cycle primaire, en vue de les familiariser à la nature de la dyslexie et aux différentes stratégies de 
compensation. De plus, il s'avère indispensable d'accomplir d'autres études visant à développer la conscience des enseignants des langues maternelle ou étrangère à propos des autres troubles d'apprentissage tels que la dysgraphie et la dysphasie. Enfin, une étude longitudinale, sur environ une année scolaire, semble importante pour juger l'efficacité des stratégies utilisées auprès des dyslexiques sur le développement de leur capacité à lire ou à orthographier.

\section{Bibliographie}

Aghababian, V. et Nazir, T. (2007). Modèle et évaluation de la lecture et des dyslexies chez l'enfant: apport de l'effet de la position optimale du regard dans le mot. In E. Demont et M$\mathrm{N}$ Metz-Lutz (Eds), L'acquisition du langage et ses troubles (pp.81-124). Marseille: Solal.

Arseneault, S. et al. (2009). The dyslexia handbook for teachers and parents in South Dakota. Départment d'éducation. Récupéré du site: http://doe.sd.gov/oess/documents/ SPED DyslexiaGuide.pdf.

Association canadienne de la dyslexie. (S. D.). Qu'est-ce que la dyslexie? Récupéré du site: http://www.Dyslexia association.ca/francais/questce.shtml.

Association canadienne des troubles d'apprentissage (ACTA). (2002). Définition nationale des troubles d'apprentissage. Récupéré du site: http://www.ldac-acta.ca/fr/en-savoirplus/d\%C3\%A9finition-des-tas/d\%C3\%A9finitionofficielle-des-tas.

Association internationale de la dyslexie. (2012). Just the facts ... Récupéré du site: http://www.interdys.org.

Association internationale de la dyslexie à Ontario (2002). Research definition of dyslexia. Récupéré du site: http:// www.idaontario.com.

Association québécoise pour les enfants dyspraxiques. Récupéré du site: http://www.dyspraxie-aqed.ca/dyspraxie-aqed 008. $\underline{h t m}$. 
Augade, L. et Thyss, M. (2000). Essai de dépistage des enfants dyslexiques par les enseignants au primaire. I.U.F.M. de l'Académie de Montpellier Centre de Mende. Récupéré du site: https://www.fichier-pdf.fr/2012/05/12/essai-dedepistage-de-dyslexie-par-les-enseignant/.

Bessac, J. (2013). Troubles des apprentissages: Dictionnaire pratique. Paris: Tom Pousse.

Brazeau-Ward, L. (2003). Quelle confusion! Est-ce de la dyslexie ou un trouble d'apprentissage? Bibliothèque nationale du Canada. Ottawa: Centre canadien de la dyslexie.

Chaix, Y., Trabanino, M., Taylor, M. et Demonet, J. - F. (2005). La dyslexie développementale: apports récents de la génétique et de la neuro - imagerie. In C. Hommet, I. Jambaqué, C. Billard et P. Gillet (Eds.). Neuropsychologie de l'enfant et troubles du développement (pp. 73-104). Marseille: Solal.

De Partz, M. P. et Valdois, S. (2000). Troubles du langage et intervention: Les dyslexies et dysorthographies acquises et développementales. In: J. Rondal et S. Xavier (Eds.). Troubles du langage: bases théoriques, diagnostic et rééducation (pp. 749-795). Liège: Mardaga.

Dejonckère, L. (2013). Les adaptations pédagogiques dans l'apprentissage de la lecture pour l'élève dyslexique, en CM2, en mettant l'accent sur la prise en charge des difficultés de compréhension (Thèse de maîtrise non publiée). Institut Universitaire de Formation des Maîtres. Ecole interne de l'Université d'Artois.

Délitroz, M. et al. (S.D.) Dyslexie, dysorthographie ... ... que faire quand cela dysfonctionne? Un mémo du Canton du Valais à l'intention des enseignants. Récupéré du site: http://www.adsr.ch/index.php/fr/component/.../1-textesde-lois.html?

Denis, L. (2009). Understanding dyslexia: a guide for teachers and parents (1e edition). Maidenhead: Open university press. 
Dickie, C., Ota, M. and Clark, A. (2013). Revisiting the phonological deficit in dyslexia: Are implicit nonorthographic representations impaired? Applied Psycholinguistics, (34), 649- 672.

Direction générale de l'enseignement obligatoire (DGEO). (2008). Dyslexie et dysorthographie: informations et recommandations à l'usage des enseignants. Département de la formation de la jeunesse et de la culture. Récupéré du site:http://www.vd.ch/fileadmin/...pdf/Brochure Dyslexie .pdf.

Doan, M. (2014). Sensibilisation: les TSL. Adaptation scolaire et scolarisation des élèves en situation de handicap. Formation des auxiliaires de vie scolaire. Rouen Le Roman. Récupéré du site:http://www4.ac-nancy-metz.fr/ia54-gtd/

handiscolash/sites/handiscolash/IMG/pdf/ANIMATION dy slexie avs 2013 -2014-2.pdf.

Faire un test de Wilcoxon sur deux échantillons appariés avec XLSTAT. Récupéré du site: http://www.xlstat.com/fr/ centre-d-apprentissage/tutoriels/12.html.

Felber, S. et Michel, J. (2012). Les aménagements pédagogiques mis en place pour les élèves présentant une dyslexie: points de vue des enseignant-e-s et des élèves (Thèse de maîtrise non publiée). Université de Genève.

Golliet, G. (2011). La dyslexie, prise en charge à l'école et à la maison (Tome 1). Récupéré du site: http://www.dyscoaching.com/image/telechargement/tome1-webechantillon.pdf.

Habib, M. (2002). Aspects étiologiques des dyslexies. In: Cheminal, R. et Brun, V. (Eds.). Les dyslexies (pp. 4-22). Paris: Masson.

Habib, M. (2014). La constellation des dys: bases neurologiques de l'apprentissage et de ses troubles. Paris: de Boeck.

Habib, M., Giraud, K., Rey, V. et Robichon, F. (2003). Neurobiologie du langage. In: J. Rondal et X. Seron. (Eds.). 
Troubles du langage: bases théoriques, diagnostic et rééducation (pp. 11- 55). Belgiques: Mardaga.

Hunziker, K. et Pulzer-Graf, P. (2012). Maintenir et encadrer des élèves aux besoins particuliers dans l'école régulière: une étude de dix situations d'intégration dans des classes vaudoises. Récupéré du site: http://edudoc.ch/record/ 107280/files/zu13050.pdf.

Institut national de la santé et de la recherche médicale (INSERM). (2007). Dyslexie, dysorthographie, dyscalculie Bilan des données scientifiques, expertise collective. Paris: INSERM.

Juhel, J.- C. (1998). Aider les enfants en difficulté d'apprentissage. Les presses de l'université Laval.

Knudsen, L. (2012). Dyslexia and Foreign Language Learning (Thèse de maîtrise non publiée). Université Malmö. Récupéré du site: http://dspace.mah.se/bitstream/handle/2043/13884/Dys lexia\%20and\%20Foreign\%20Language\%20Learning\%20$\% 20$ Lina\%20Knudsen.pdf?sequence=2.

Lapierre, M. (2008). Mise au point et validation d'un programme de rééducation pour les enfants présentant une dyslexie à prédominance visuelle ou mixte (Thèse de doctorat non publiée). Université du Québec à Trois-Rivières.

Leclercq, A.-L. et Leroy, S. (2012). Introduction générale à la dysphasie: caractéristiques linguistiques et approches théoriques. In: C. Maillart et M.- A. Schelstraete (Eds). Les dysphasies: de l'évaluation à la rééducation (pp 5- 34). Paris: Elsevier Masson.

Lederlé, E. et Maeder, C. (2014). Troubles dyslexiques et anomalies de la vision. In C. Kovarski (Ed.). Les anomalies de la vision chez l'enfant et l'adolescent (pp. 373- 382). Paris: Lavoisier.

L'Internaute. Récupéré du site: http://www.linternaute.com/ dictionnaire/fr/definition/dyslexie/. 
Lyon, R., Shaywitz, S. and Shaywitz, B. (2003). Defining dyslexia, comorbidity, teachers' knowledge of language and reading: A definition of Dyslexia. Annals of dyslexia, 53 (1), 1- 14.

Marin, B. et Legros, D. (2008). Psycholinguistique cognitive: lecture, compréhension et production de textes (1e édition). Bruxelles: De Boeck université.

Martin, L. and Borkowski, B. (2014). Essentials of special education: diversity in the classroom. Florida: Green Apple Publications, Inc.

National Council for Special Education (NCSE). (2014). Children with special educational needs: information booklet for Parents. Récupéré du site: http://ncse.ie/wp-content/ uploads/2014/10/ChildrenWithSpecialEdNeeds1.pdf.

Nijakowska, J. (2010). Dyslexia in the Foreign Language Classroom. Toronto: Multilingual matters.

Ortiz, C. (2014). Les écrits des enfants dyslexiques: comment aider les élèves dyslexiques dans leur production écrite? (Thèse de maîtrise non publiée). Université d'Orléans.

Pelletier, E. et Léger, C. (2004). Les troubles d'apprentissage: Guide pour les enseignants. Récupéré du site: http://www.bdaa.ca/biblio/apprenti/aqeta/taguide/tagui de.pdf.

Ringard, J.-C. (2000). A propos de l'enfant "dysphasique" et de l'enfant "dyslexique". Récupéré du site: http://media. education.gouv.fr/file/95/7/5957.pdf.

Saint- Pierre, M.- C., Dalpé, M., Lefebvre, P. et Giroux, C. (2010). Difficultés de lecture et d'écriture: prévention et évaluation orthophonique auprès des jeunes. Presses de l'université de Québec.

Snowling, M. (2001). From language to reading and dyslexia. Dyslexia, 7, 37-46.

(2013). Early identification and intervention for dyslexia: A contemporary view. Journal of Research in Special Educational Needs, 13(1), 7- 14. 
Sprenger-Charolles, L. et Sernicales, W. (2003). Acquisition de la lecture et de l'écriture et dyslexie: Revue de la littérature. Revue Française de linguistique appliqué, 8 (1), 63 - 90.

Thorwarth, C. (2014). Debunking the Myths of Dyslexia. Leadership and Research in Education: The Journal of the Ohio Council of Professors of Educational Administration (OCPEA), 1, 51- 66.

Thoulon - Page, C. (2009). La rééducation de l'écriture de l'enfant: Pratique de la graphothérapie (2e édition). Paris: Masson.

Torgesen, J., Foorman, B. and Wagner, R. (2007). Dyslexia: A Brief for Educators, Parents, and Legislators in Florida (Technical Report No. 8) Florida Center for Reading Research, Tallahassee, FL (pp. 1- 18). Récupéré du site: http: //www.dys-add.com/resources/SpecialEd/FCRRRepton Dysl.pdf.

Valdois, S. (2005). Traitements visuels et dyslexies développementales In: C. Hommet, I. Jambaqué, C. Billard et Gillet, P. (Eds.). Neuropsychologie de l'enfant et troubles du développement (pp. 267 -300). Marseille: Solal Editeurs.

Van Hout, A. et Estienne, F. (2001). Les dyslexies: décrire, évaluer, expliquer, traiter ( $3^{e}$ édition). Paris: Masson.

Zorman, M., Lequette, C. et Pouget G. (2004). Dyslexies: intérêt d'un dépistage et d'une prise en charge précoce à l'école. Evaluation du BSEDS 5-6. In M. N. Metz -Lutz, E. Demont, C. Seegmuller, M. De Agostini et N. Bruneau (Eds), Développement cognitif et troubles des apprentissages: évaluer, comprendre, rééduquer et prendre en charge (pp. 245- 270). Solal, Marseille. 